De quelques enjeux et usages historiques du Français fondamental

\title{
Vocabulaires logiques, vocabulaires simplifiés et Français élémentaire
}

Javier Suso López

\section{(2) OpenEdition \\ Journals}

Édition électronique

URL : https://journals.openedition.org/dhfles/1195

DOI : $10.4000 /$ dhfles. 1195

ISSN : 2221-4038

Éditeur

Société Internationale pour l'Histoire du Français Langue Étrangère ou Seconde

Édition imprimée

Date de publication : 1 janvier 2006

Pagination : $97-118$

ISSN : 0992-7654

Référence électronique

Javier Suso López, «Vocabulaires logiques, vocabulaires simplifiés et Français élémentaire ».

Documents pour l'histoire du français langue étrangère ou seconde [En ligne], 36 | 2006, mis en ligne le 20 novembre 2014, consulté le 27 mai 2021. URL : http://journals.openedition.org/dhfles/1195; DOI : https://doi.org/10.4000/dhfles.1195

Ce document a été généré automatiquement le 27 mai 2021.

(C) SIHFLES 


\title{
Vocabulaires logiques, vocabulaires simplifiés et Français élémentaire
}

\author{
Javier Suso López
}

1 Le Français élémentaire (FE) se situe dans la jonction d'un double courant d'études : celui des vocabulaires de base « logiques » et celui des vocabulaires simplifiés obtenus grâce à des enquêtes de fréquence, tous deux porteurs d'une mentalité nouvelle sur la question du lexique dans l'enseignement d'une langue.

\section{Vocabulaires logiques et vocabulaires simplifiés}

2 Deux voies de recherche paraissent être tracées dès le début: la simplification y est faite soit par des procédés rationnels ou logiques (c'est le cas du Basic English - British American Scientific International Commercial ${ }^{1}$ - élaboré par C.K. Ogden et I.R. Richard en 1928, et du Defining Vocabulary de M. West de 1929), soit par des procédés mettant en œuvre des méthodes statistiques (Thorndike 1921; Henmon 1924 ; Vander Beke 1927 ; Baker 1928, Haygood 1937, etc.). L'application de ces outils ne va pas concerner seulement l'enseignement d'une langue étrangère ; certains auteurs voudront tirer parti de ces vocabulaires simplifiés pour améliorer à leur tour l'enseignement de la langue maternelle (c'est le cas des ouvrages de Aristizabal, de 1938, ou de Dottrens et Massarenti de 1948²).

3 Dans ce contexte, quelle est la particularité du Français élémentaire (1954)? Quant à la méthode utilisée, il se situe à l'intérieur du deuxième courant de recherche; mais, il partage avec le Basic English une même fonction centrale, c'est-à-dire, l'enseignement rénové de la langue étrangère. Ceci dit, les réalisateurs du FE (1956) mettent en relief leurs distances par rapport aux recherches antérieures : tant du côté des propositions antérieures de français de base que, surtout, du côté du Basic English.

4 Le jugement très négatif porté sur le Basic English par les réalisateurs du FE s'explique par le besoin de prendre des distances à son sujet, à cause du «tollé » qui s'était formé à l'annonce du projet de création d'un français de base ${ }^{2}$. Lors de l'annonce par le ministre de l'Éducation nationale de l'époque, l'initiative FE avait été rapprochée du 
Basic quant à ses fonctions ("la diffusion la plus large possible d'un vocabulaire suffisant ", 1956: 12). Les critiques furibondes et acharnées, venant de tout bord, obligeront les auteurs du FE à préciser que ce n'était nullement un Basic français ${ }^{3}$. Le Basic English est ainsi pour les auteurs du FE une langue « fermée » (1956, p. 12) :

« Une langue semi-artificielle conçue par C.K. Ogden et I.R. Richards dans les années vingt. Il s'agit, en s'appuyant sur les réseaux synonymiques de la langue anglaise, de la réduire aux termes minimaux (premiers) permettant de définir (et donc de comprendre et de s'exprimer dans une certaine mesure) tous les autres termes de la langue, un peu comme dans un dictionnaire. Le Basic est donc un vocabulaire minimal à portée théorique maximale formant un tout, c'est une 'langue universelle à base d'anglais [...] pouvant se suffire à elle-même. » $(1956: 28)^{4}$

Personne ne met en doute aujourd'hui que le Basic a signifié la constitution d'un formidable outil pour l'enseignement (et donc la diffusion) de l'anglais à des millions d'élèves de toutes sortes (dont surtout des adultes, des soldats, des professionnels) dans les années 1930 et 40, ce qui le situe au rang des principales réussites de la linguistique appliquée (et nous employons cette expression avant la lettre de façon délibérée). Audelà de ses faiblesses, corrigées dans les éditions postérieures, et surtout dans les applications pédagogiques qui lui font perdre de sa « rigidité », le Basic est devenu « un moyen efficace de diffusion de la langue anglaise » dans tous les pays du monde, et pas seulement dans les pays colonisés par l'Angleterre, comme le reconnaissent les auteurs du FE (1956, p. 25). Ceux-ci reproduisent une lettre de M.S.B. Wynburne, professeur à Belfast, où il manifeste avec enthousiasme les bienfaits de cet outil, ainsi que son désespoir quand il constate qu'il n'existe aucun matériel similaire pour le français :

«J'ai eu l'occasion, pendant la guerre, d'enseigner le Basic English à des troupes hindoues, j'ai pu disposer du matériel admirable mis à la disposition de l'étudiant. Je suis incapable maintenant, bien que spécialiste de français, d'enseigner votre langue sans éprouver le plus vif désespoir quand j'oppose chaque jour la présentation du matériel linguistique au petit bonheur, l'immensité du vocabulaire et des constructions, que je signale en vain au passage, et d'autre part l'enseignement méthode exhaustif de notre langue que je viens de donner.» (Gougenheim et al. $1956: 25$ ).

Quant à l'étude de Michael West (1929), nous relevons uniquement sa conclusion : il prouve qu'avec un vocabulaire de 1072 mots anglais il est possible de traduire le premier tome de Monte Cristo (qui possède environ 50000 mots). Il publie lui-même un roman avec un lexique ne comportant que 1779 mots différents, et défend qu'un vocabulaire de cette étendue comprend tous les termes indispensables (in Bénédict \&Humbert 1950, p. 203).

7 La constitution de vocabulaires logiques n'était pas la seule voie de recherche. Au contraire, dès le début du siècle, certains chercheurs ou professeurs s'intéressent à la question des vocabulaires standardisés ou réduits grâce à des procédés statistiques (le word-count : tri et décompte lexical) dans l'enseignement des langues vivantes, et des vocabulaires simplifiés sont proposés pour les langues anglaise, française, espagnole ou allemande (Gougenheim et al. 1956, chapitre premier). L'honneur de la première étude concernant l'établissement d'un choix lexical revient à l'allemand J.W. Käding, qui publie un Häufigkeitswörterbuch der deutschen Sprache en 1898, réalisé grâce à la collaboration d'étudiants sténographes allemands et destiné à l'enseignement de la sténographie. Cette étude n'aura point de répercussion sur l'enseignement de la langue allemande (maternelle ou étrangère). Mais c'est aux États-Unis et au Canada que ces 
études prennent corps de façon particulièrement importante : c'est par ces pays que nous allons commencer notre présentation.

\section{2. Études et propositions réalisées aux États-Unis et au Canada}

James D. Haygood estime que les recherches sur le vocabulaire entreprises aux ÉtatsUnis au début $\mathrm{du} \mathrm{XX}^{\mathrm{e}}$ siècle partent de la constatation des défauts de l'enseignement des langues étrangères de la part du rapport du «Comité des Douze» de la Modern Language Association of America, qui fut publié en $1898^{5}$. Ce rapport, que Haygood compare à la brochure publiée par W. Viëtor en 1882, eut l'effet de «servir de base à des investigations ultérieures d'où sortirent toutes sortes de soi-disant méthodes, plus ou moins sérieuses " ([1937]1948², p. 1). Le fait est que les professeurs de langues étrangères des États-Unis s'engagent dans une voie de recherche nouvelle, qu'on pourrait cerner grâce à la question suivante formulée par Haygood : « Combien de mots [français, dans notre cas] doit connaître un élève pour pouvoir lire et comprendre un texte français de difficulté moyenne ? Quels sont ces mots ? "([1937]1948², p. 3).

De 1918 à 1923, trois articles, publiés tous trois dans The Modern Language Journal, vont s'occuper de cette question (voir Annexe 1). Tout d'abord, en 1918, E. W. BagsterCollins :

- « déplore le manque de standardisation dans l'étude des vocabulaires étrangers ; il y voit la cause essentielle de la médiocrité des résultats obtenus. Pour la confection de ces vocabulaires, il exige une sélection soigneuse et rationnelle des mots »; - en 1919, John C. Waigel signale les «profondes divergences qui existaient dans la conception d'un vocabulaire idéal pour les cours de langues vivantes de première et de deuxième année »; en 1923, Marie V. Keller

- expose les résultats d'une enquête faite en 1911 à Depauw University, en vue de préciser le nombre de mots que possédait dans sa langue maternelle une étudiant novice. Ce nombre allait de 2970 à 4560 mots. Chose remarquable, Miss Keller déduit de ces statistiques que 2000 mots étrangers, choisis scientifiquement, pourraient suffire au vocabulaire d'un bachelier de high school pour la lecture d'une langue étrangère. (in Haygood [1937]19482, p. 4)

Les choses vont aller désormais très vite: nous allons signaler tout d'abord deux réalisations au sujet des vocabulaires simplifiés pour la langue anglaise, qui ont marqué l'histoire de ce domaine de recherche et sur lesquels vont s'inspirer les propositions pour le FLE. Tout d'abord, le Teacher's Word Book de E.L. Thorndike (1921), qui présente 10000 vocables différents, de haute fréquence, choisis dans un total de quelque 4500000 mots extraits de livres de lecture d'une extrême variété. «La valeur de ce calcul est sérieusement diminuée par le fait qu'il ne prend pas en ligne de compte les homonymes; c'est-à-dire qu'aucune distinction n'est faite entre les mots d'orthographe semblable mais différents de sens» (Haygood [1937]1948², p. 9). Il aura une faible répercussion pour l'enseignement de la langue anglaise, puisqu'il fut bientôt remplacé par le Basic English. Quant à H.E. Palmer, nous nous limitons à reprendre le commentaire réalisé par les auteurs du FE à son sujet :

« Nous ne ferons que mentionner la méthode de H.E. Palmer, dont M.H. Bongers [...] est le disciple enthousiaste. H.E. Palmer, qui a dirigé l'Institute for Research in English Teaching à Tokyo, a dressé des listes de vocabulaire anglais (The 600 English Words for Class-room Vocabulary, Tokyo, IRET ; The 500 english Words of most frequent occurrence, Tokyo, IRET, 1931; The second English Words of most frequent occurrence, 
Tokyp, IRET, 1931 ; et, avec, A. S. Honrby, Thousand Word English, London, 1937). Les listes de H.E. Palmer sont établies empiriquement, mais soumises à posteriori à une vérification méthodique d'après le pourcentage de compréhension obtenu." (Gougenheim et al. $1956: 26$, note 6)

11 En passant maintenant au domaine de la langue française en tant que langue étrangère, on est surpris de constater que, longtemps, les auteurs qui s'engagent dans la voie d'améliorer l'enseignement du FLE à travers les vocabulaires simplifiés sont des étrangers (voir Annexe 1): il s'agit des américains V.C.A. Henmon (1924), Ben D. Wood (1927), M. Vander Beke (1927), F. Baker (1928), F.D. Cheydleur (1930), James Douglas Haygood (1937), et J.B. Tharp (1939) ; du suédois M.B. Schlyter (1951); de l'hollandais L. Verlée (1954). Il faudra attendre le Français élémentaire (1954) pour que des auteurs français prennent la relève de ces travaux.

C'est V.C.A. Henmon, professeur à l'Université de Wisconsin, qui va faire un pas important dans la direction de constituer un vocabulaire simplifié pour la langue française tout en dépassant les méthodes empiriques employées au début du siècle. Il constate lui aussi que les inventaires « publiés à l'intention des commençants étaient en désaccord complet sur le vocabulaire qu'ils proposaient » (Gougenheim et al. 1956, p. 28), puisqu'ils étaient fondés sur l'intuition ou l'expérience professionnelle des auteurs de manuels: ils étaient donc difficilement généralisables, puisqu'ils étaient "dépourvus d'objectivité " et ne "sauraient être considérés comme un lexique rationnel et scientifiquement compilé » (Bénédict, Humbert 1950, p. 200) :

«Chaque auteur emplit empiriquement ses ouvrages du vocabulaire qui lui a paru le plus utile, mais la comparaison des divers primers (syllabaires) en usage dans nos classes est, à ce point de vue, bien instructive. On y voit que tel mot, considéré par tel auteur comme indispensable (puisqu'il l'emploie dès les premières leçons), ne figure nulle part dans les ouvrages de ses confrères! Et des mots courants, des expressions d'un usage quotidien sont arbitrairement laissés de côté.» (Roger 1931)

Henmon « résolut [ainsi] d'établir scientifiquement quels étaient réellement en français les mots les plus usuels et de déterminer leur degré de fréquence " (Gougenheim et al. 1956, p. 28). Il prend ainsi comme base de travail des textes appartenant à la littérature française de la seconde moitié du XIX ${ }^{e}$ siècle, donne à plusieurs professeurs du secondaire qui collaborent avec lui des tranches de cinq mille mots tirés d'un ou de plusieurs textes, grâce auxquels il établit un relevé de 400000 mots. Dans la publication finale (1924), les mots sont disposés en deux listes : la première est disposée par ordre alphabétique avec l'indication de la fréquence en regard de chaque mot; la deuxième est disposée par ordre de fréquence. Cependant, la liste de mots de Henmon néglige le « range » des mots (répartition, nombre de textes dans lesquels on trouve chacun des mots), ainsi que les différents sens d'un même mot, ce qui va affaiblir son intérêt scientifique.

14 Les ouvrages de Thorndike et de Henmon produisent l'effet de réveiller de nombreux professeurs aux États-Unis au besoin de changer des pratiques traditionnelles au sujet de l'apprentissage du vocabulaire. De nombreuses études et propositions de vocabulaires simplifiés apparaissent aux États-Unis. Nous présentons dans l'Annexe 1 une relation de ces travaux.

Nous allons nous occuper tout d'abord du vocabulaire proposé par Florence M. Baker (1928). Fl. Baker constate tout d'abord l'absurdité de la façon habituelle d'apprendre une langue, à savoir, à l'aide d'un manuel qui contient un texte de lecture, beaucoup de grammaire, et une série d'exercices de traduction de la langue maternelle au français, 
où l'élève est confronté à une "collection of words varying in number from approximately twelve hundred to fifteen undred. These words are chosen arbitrarily by the writer of the text-book and rarely conform to those selected by other text-book writers » (1928, p. 369). Contre cette façon de faire, une idée s'ouvre de plus en plus le chemin des esprits et des mentalités, selon laquelle ce n'est pas parce que l'élève apprend plus de mots qu'il acquiert effectivement un vocabulaire plus vaste : " we shall some day abandon the idea that exposing the student to a large number of words necessarily means that he acquieres a large vocabulary ». Elle met en avant ainsi la notion de vocabulaire passif, utilisé lors de la compréhension (que l'on reconnaît), qui est plus large que le vocabulaire actif (que l'on connait), utilisé effectivement lors de l'expression. Il faut laisser de côté cette matière morte (« dead wood») et se limiter ainsi à faire apprendre aux élèves de première année 400 mots, mais d'une manière effective, en faisant qu'ils reviennent continuellement dans les textes et les exercices.

Comment choisir ces 400 mots ? À la méthode des "séries ", elle préfère le critère de la fréquence. Elle s'appuie sur les propositions de Thorndike (pour la langue anglaise) et de Henmon (pour la langue française). Mais elle corrige les données que la fréquence fournie en brut, et les complète avec le critère de l' " association » : elle se rend compte que les listes de ces auteurs contiennent - parmi les 400 mots les plus fréquents - trop de conjonctions, adverbes et prépositions, ou du moins beaucoup plus qu'il n'en faut pour construire des phrases simples. Elle en retranche donc, mais aussi elle en rajoute, après observation, pendant trois ans, des compositions de jeunes écoliers en français et en anglais, et des conversations et des remarques qui surgissent lors de l'exercice de la conversation française. Elle complète ainsi de façon empirique les pures données de la fréquence, estimant que ce seul critère fournit un outil incomplet et non adapté aux besoins de la communication scolaire.

17 Ainsi, elle arrive à la conclusion que certaines séries sont nécessaires : c'est le cas des nombres (de un à vingt, même si quatorze ou seize ont un degré de fréquence insuffisant) ; c'est aussi le cas des jours de la semaine, des mois de l'année. Dans ces cas, c'est la série entière qu'il faut connaître. D'autre part, les mots de la classe sont nécessaires pour engager bon nombre de dialogues (professeur, encre, crayon, bureau, etc.). D'autres additions servent à compléter des questions tenant à la culture française : un franc, un centime, un sou; ou encore des domaines restés incomplets dans les listes de Thorndike et Henmon : c'est le cas de fruit, beurre, gâteau, sucre, sel... (Baker 1928, p. 372-373).

En résumé, l'expérience mise en oeuvre par Fl. Baker contient bien de leçons. C'est à travers la distinction entre vocabulaire actif et vocabulaire passif qu'elle arrive à la conclusion du besoin d'établir un vocabulaire réduit, et elle met cette distinction au cœur de sa sélection de vocabulaire. Elle est bien consciente que la liste qu'elle propose est « arbitraire » $(1928$, p. 373), mais nous avons là un précieux outil à moitié chemin entre le critère de fréquence (sur deux langues différentes), les observations directes sur l'oral de la classe, et les constatations empiriques issues des devoirs de composition des écoliers (en deux langues également). Elle voit parfaitement les limites de la méthode statistique pure par fréquence; sa décision de retrancher des mots grammaticaux et de compléter la liste par des associations liées aux situations vécues par les écoliers (et en cela, elle se démarque de la méthode des séries) elle annonce la notion de disponibilité utilisée par les auteurs du FE. Finalement, la liste qu'elle propose n'est qu'une initiation à la langue française, que l'élève poursuivra les années 
postérieures. Mais l'avantage qu'elle offre à ses yeux est celle de constituer le socle de mots de base, que les élèves devront acquérir effectivement, en laissant de côté d'autres autres mots qui peuvent apparaître (dans les textes de lecture), mais dont il suffira de reconnaitre ou de comprendre à cette occasion-là :

"These are words which will fit without artificial arrangement into the conversation, reading, or composition. They will also serve to illustrate grammar rules when necessary. This does not, however, claim to be an exclusive list. There is no thought that the student will be expected to see only these words during his first year. There is no raison why he should not use a dictionary, a newspaper, or French in any form in which it offers itself. But il is felt that these are the words on which to concentrate. » $(1928: 376)$

Avec l'ouvrage de George E. Vander Beke $(1927)^{6}$, une barrière est franchie : ce ne sont plus des recherches ou des propositions de professeurs individuels, ce sont maintenant des investigations conduites sous les auspices des prestigieuses associations Americain and Canadian Commitees on Modern Languages, dont les travaux concernent également les vocabulaires fondamentaux pour l'espagnol (1927), pour l'allemand (1928) et pour le portugais brésilien (1945). La finalité d'améliorer l'enseignement des langues vivantes est clairement posée dans ces initiatives.

Le Word Book de Vander Beke (1927) surgit ainsi du désir de « renouveler la matière de l'enseignement des langues modernes sur une base objective ». Ces Comités confient à George E. Vander Beke, professeur à l'université Marquette, la tâche de reprendre le dictionnaire d'Henmon pour l'améliorer. Le dictionnaire de Vander Beke, tel qu'il est publié, comprend trois parties: la première est la liste des mots très fréquents de Henmon; la deuxième présente les mots par ordre de range décroissant (6 067 mots sont ainsi classés) ; la troisième partie présente les mots des deux premières par ordre alphabétique. Les perfectionnements apportés relèvent de quatre points :

- le nombre de mots contrôlés (1 547 748, y compris les 400000 comptés par Hemmon) : il établit ainsi un inventaire de 6067 mots ;

- «la grande variété de sources utilisées pour le recensement » (Bénédict, Humbert 1950, p. 202) : il introduit des textes scientifiques, des textes de journaux et de périodiques des années vingt ; des pièces de théâtre ; il élimine les textes en vers...

- la distinction des emplois du mot. Vander Beke distingue les homonymes homographes (ce que Henmon faisait dans certains cas), tels que

- -son (mot grammatical, possessif) et son (substantif : le son de la cloche)

- -mal (adverbe) et mal (substantif)

- -louer (exalter, glorifier) et louer (donner à loyer)

- -pêche (fruit) et pêche (action de pêcher)

- -le manche et la manche

- -le livre et la livre

- la distinction des emplois grammaticaux d'un même mot :

- -haut (adverbe) et haut (adjectif)

- -se souvenir (verbe) et souvenir (substantif)

- -fort (adjectif) et fort (adverbe)

- -devant (adverbe) et devant (substantif)

- -la distinction, dans certains cas, des différentes acceptions d'un même mot (polysémie) :

- -gauche (opposé à droite) et gauche (maladroit)

- -pousser (croître) et pousser (faire effort en poussant) (in Gougenheim et al. 1956 : 29-31). 
listingue pas cependant, par exemple, plume (à écrire) et plume (d'oiseau), fille (terme de parenté) et jeune fille, ou encore ouvrier et ouvrière (tandis que sont notés séparément duc et duchesse, prince et princesse, etc.).

« l'innovation la plus importante, par rapport à Henmon, est d'avoir ajouté à la considération de la fréquence celle de la répartition (range). Il estime, en effet, qu'un mot qui se rencontre 1 fois chez 5 auteurs différents est plus important qu'un mot que se rencontre 10 fois chez un seul auteur » (Gougenheim et al. 1956, p. 31).

«Ainsi, le range du mot heure est bien lus étendu que celui du mot écho, puisque le premier figure dans quasi tout ouvrage français; le second, en revanche, n'y apparait que dans un nombre fort limité. Évaluation indispensable, car si l'on veut donner une image exacte de l'utilité relative d'un vocable, il faut considérer à la fois sa fréquence et son range » (Bénédict, Humbert $1950: 202)$.

Vander Beke élimine du relevé les 69 premiers mots du Word Book de Henmon, c'est-àdire les mots qui avaient une fréquence supérieure à 450 . Il semblait incontestable que ces mots étaient indispensables, et un nouveau relevé augmentait considérablement la tâche des dépouilleurs. Ce sont surtout des mots grammaticaux (les articles, les déterminatifs, les pronoms, certains adverbes, les verbes auxiliaires avoir et être), mais aussi 5 adjectifs (autre, bon, grand, petit, tout), 4 noms (enfant, femme, homme, jour), et 10 verbes (en plus d'être et avoir : aller, dire, donner, faire, pouvoir, prendre, savoir, venir, voir, vouloir).

L'enquête lexicologique de Vander Beke devient ainsi un outil de travail incontournable le long des années 1930 et 1940, comme le reconnaissent Bénédict et Humbert : « le fait que la plupart des manuels d'enseignements du français, publiés de nos jours aux EtatsUnis, se réfèrent à sa liste est une preuve éclatante de sa validité » $(1950$, p. 202). Les auteurs du FE remarquent que, même si la «méthode peut [...] être considérée comme valable», certains défauts importants sont relevés: «le dépouillement fondé uniquement sur la langue écrite ", "le caractère littéraire et archaïque du vocabulaire ainsi obtenu ", et un dernier aspect, à savoir la "faible fréquence des noms concrets par rapport aux verbes» (Gougenheim et al. 1956, p. 36), question qui est une " constante de toutes les recherches de fréquence». à Londres (1935), et où participent des linguistes (entre autres, ceux qui avaient travaillé au développement des vocabulaires simplifiés, tels que M.West, H. Palmer, P. West), des psychologues (tels que E.L. Thorndike), et des pédagogues et des responsables politiques éducatifs (tels que L. Faucett, R.H. Fife ...), avait montré l'importance que la question des vocabulaires simplifiées avait acquise outreAtlantique. C'était tout d'abord un outil de communication entre populations parlant des langues différentes (ou "lingua franca», selon l'expression utilisée dans le Rapport), pour des touristes et des voyageurs, mais ils possédaient aussi un intérêt éducatif, un point de départ susceptible d'être enrichi par la suite selon des orientations stylistiques, culturelles ou autres.

Les travaux concernant les vocabulaires simplifiés continuent ainsi de se produire: James B. Tharp (1939) utilise dans sa liste de 3340 mots plusieurs sources, dont celles de Henmon et Vander Beke. Son œuvre comprend trois parties : a) « une liste alphabétique donnant le mot français, sa prononciation notée en alphabet phonétique, le sigle du groupe et du sous-groupe de fréquence auquel il appartient, la catégorie grammaticale et sa traduction, ainsi que la traduction de diverses expressions où ils figurent »; b) un classement par groupement de fréquence; c) une liste classée par catégories 
grammaticales (Gougenheim et al. 1956, p. 40-41). Nous mettons en relief pour notre part la réunion de la liste de fréquence avec la notation phonétique de l'API comme l'une des idées remarquables de cet auteur.

\section{3. Études et propositions réalisées en Europe}

\subsection{Applications pour le français langue étrangère}

Nous allons passer maintenant à la présentation des ouvrages réalisés en Europe. Nous commençons tout d'abord avec les études visant une application pour le français langue étrangère (FLE). James Douglas Haygood ([1937] 1948) ${ }^{7}$ va effectuer une recherche inspirée de celle de $\mathrm{M}$. West et de la thèse de $\mathrm{M}$. A. Emery : vérifier si la liste établie par Vander Beke est vraiment utile à la lecture d'une série d'œuvres utilisées dans les premiers cours de FLE. Il arrive à la conclusion que l'inventaire intégral de Vander Beke (plus de 6000 mots, comme nous l'avons dit plus haut) est trop vaste pour constituer un vocabulaire fondamental. Il sélectionne dans la liste de Vander Becke les mots qui réunissent certaines conditions (présenter un intérêt certain; être de difficulté moyenne; être de longueur moyenne ; être tirés d'une éditions originale), puis il s'adonne lui-même à des travaux de recensement, qu'il décrit le long de sa thèse, basés sur le principe d'écarter tout élément subjectif. La première conclusion de son travail est la suivante :

«Les 69 mots de la première partie et les 2000 premiers mots de la seconde partie $\mathrm{du}$ French Word Book nous paraissent une base suffisante permettant de lire les textes français utilisés au cours du premier cycle d'études françaises, ainsi que la plupart des romans ou d'autres œuvres littéraires françaises de moyenne difficulté qui ne sont pas destinées spécialement à l'enseignement de la langue.» ([1937]19482:46)

D'autre part (il s'agit de sa seconde conclusion), son étude corrobore la liste de Vander Becke «sur les mots inclus et exclus, et sur l'ordre dans lequel sont placés les premiers» (id., 47). Malgré l'existence de quelques défauts, l'enseignement à tirer de son étude est évident : « au lieu de déplorer ces défauts et critiquer l'œuvre de Vander Becke, servons-nous de la liste le plus possible pour résoudre les problèmes de vocabulaire qui nous intéressent» (id., 47-48). L'intérêt de l'ouvrage de Haygood dépasse de beaucoup ces conclusions: dans son livre II (p. 49-117), il propose une "Étude pratique sur l'enseignement des langues vivantes ", où il jette les bases d'une véritable science didactique, composée de quatre points : 1 . La théorie. 2. Le matériel d'enseignement. 3. La méthode proposée. 4. Le programme d'études proposé. La démarche de travail est résolument "scientifique » : le premier élément à considérer c'est «l'ensemble des faits établis par l'expérimentation en laboratoire» (id., 49); l'insistance sur les "faits vérifiés par la pratique »; et le besoin de s'appuyer sur " des faits déterminés expérimentalement». Il annonce ainsi une nouvelle phase (ou paradigme) dans la recherche sur l'enseignement des langues vivantes.

Pour compléter ce panorama, il faut faire référence aussi à d'autres auteurs : JacquesGabriel Cahen (1946) montre que deux mille mots (et non pas six cents, comme le disent Dottrens et Massarenti dans l'Introduction de leur ouvrage, $1948^{2}$ ) ont suffi à Racine pour composer les dix-huit mille vers de ses tragédies; Börje Schlyter établit un vocabulaire de base de 2500 mots (1951), qui s'inspire nettement de celui de J.B. Tharp : ils sont classés par ordre alphabétique, avec indication également du sigle du groupe de 
fréquence, d'indications grammaticales, d'une traduction en suédois et d'indications de flexion ; L. Verlée, auteur hollandais, établit une liste de 3000 mots (1954), fondée sur la liste de Vander Beke, à laquelle il ajoute les résultats d'une enquête personnelle.

\subsection{Applications pour le français langue maternelle}

29 Nous allons parler finalement des vocabulaires simplifiés créés en vue de leur utilisation dans l'enseignement du français langue maternelle (FLM) qui présentent des considérations intéressantes sur la question. Tout d'abord, A. Prescott, qui effectue une étude sur le "vocabulaire des enfants genevois", publiée en 1923, ainsi qu'un Vocabulaire édité par le Département de l'Instruction publique de Genève (s.a.). Prescott établit une liste de 3470 mots, en recueillant le vocabulaire des enfants genevois grâce à la «technique des associations libres » : M.F. Dubois - dont nous parlerons un peu plus loin - critiquera cette méthode en constatant que cette méthode privilégie les objets qui entourent l'enfant et que « le fait de devoir écrire les mots qui l[eur] passent par la tête pendant quinze minutes place [les enfants] dans une situation anormale et étrange »(Dottrens, Massarenti 1948², p. 5).

Aussi, la liste de M. Aristizabal (1938), qui « fait partie d'un ensemble de recherches que des pédagogues belges, sous la direction de $\mathrm{M}$. Buyse, professeur à l'Université de Louvain, ont effectuées en vue de rendre plus efficace l'apprentissage de la langue et spécialement de l'orthographe (dans les écoles de langue française) » (Gougenheim et al. 1956, p. 46). Il établit un "vocabulaire mixte, contenant à la fois le vocabulaire des enfants et celui des adultes", à partir de textes écrits très variés (des lettres, des rédactions, des historiettes). Au total, il «a travaillé sur 5.525 textes, fournissant 460.727 mots. Il a relevé 12.038 mots différents, dont 4.329 avaient une fréquence supérieure à 10 " (id., p. 46). Cela explique que ce soit un vocabulaire enfantin et scolaire : la fréquence de mots utilisés par les enfants ou bien appartenant à l'école y acquièrent un taux de fréquence très élevé (c'est le cas de jouer, amuser, enfant, poupée, jouet ; ou de : école, classe, leçon, maîtresse, etc.).

31 À Louvain encore, F. Dubois reprend le travail d'Aristizabal et publie en 1939 une étude intéressante : l'Échelle d'orthographe française. Cette étude présente, pour Dottrens et Massarenti, deux grands avantages : le premier est qu'il fournit un 'vocabulaire mixte' " c'est-à-dire, constitué à partir du vocabulaire des enfants eux-mêmes, et donc "approprié à ses besoins d'expression", mais aussi, à partir de lettres d'adultes (écrivains célèbres, correspondance commerciale, lettres familières, lettres de circonstance...), donc " en prévoyant dans la mesure du possible les besoins futurs " (Aristizabal, in Dottrens, Massarenti $1948^{2}$, p. 5). Dubois élimine certains termes (ceux à faible fréquence, emplois particuliers, une série de termes religieux) et établit une liste finale de 3680 mots. Les mots retenus sont répartis en 123 listes de 30 mots chacune, " chaque mot étant inséré dans une phrase simple et courte qui en éclairait le sens et empêchait les confusions possibles. Chaque série comprenait des noms, des verbes et des adjectifs en parts égales » (Dottrens, Massarenti 1948², p. 6).

Le deuxième avantage du travail de Dubois est qu'il fournit une " gradation en difficulté d'écriture de ces mots et par conséquent la possibilité d'établir une échelle d'orthographe d'usage » (Dottrens, Massarenti 1948², p. 7). Pour cela ils dictent les mots dans de différentes écoles (386 écoles), comprenant un total de plus de 57000 élèves (garçons et filles presque à égalité), donnant un total de 1717000 graphies à corriger. 
Selon le degré de réussite obtenu, Dubois répartit les mots selon les différents degrés de l'école primaire, estimant que les mots les plus faciles sont ceux dont la fréquence (quant à la réussite) est la plus élevée.

Nous arrivons finalement au Vocabulaire fondamental du français. Contribution à un enseignement rationnel de l'orthographe d'usage $\left(1948^{2}\right)$, élaboré à Genève par Robert Dottrens et Dino Massarenti ${ }^{8}$. "Les auteurs ont confronté les listes de Dubois (Échelle d'orthographe française, 1939), qui repose sur Aristizabal ; de Haygood [...], qui comprend les 2.000 premiers mots de Vander Beke; de Prescott [...] qui comprend 3740 mots; enfin d'un vocabulaire simplifié établi par Dottrens lui-même quelques années auparavant d'après les listes de vocabulaire en usage dans les écoles du canton de Genève » (Gougenheim et al. 1956, p. 49) ${ }^{9}$. Comme le sous-titre le fait voir, le but de l'ouvrage est encore axé sur la maîtrise de l'orthographe en FLM, mais aussi ils ouvrent la porte à la question de l'apprentissage du sens des mots. Les auteurs posent trois questions initiales :

«1. Combien de mots un élève peut-il acquérir au cours de sa scolarité, tant au point de vue du sens que de l'orthographe?

2. Quels sont ces mots?

3. Peut-on connaître le degré de difficulté orthographique de chacun de ces mots, afin d'établir une échelle graduée correspondant aux différents âges ?» (1948:2)

En laissant de côté les aspects techniques (touchant à la méthode statistique) de leur travail (exposés brièvement le long des pages 3 à 14 de leur étude, où ils affirment avoir utilisé les travaux de Dubois, d'Aristizabal, de Haygood, de Dottrens lui-même, auxquels ils ajoutent le Petit Larousse illustré, dont ils confrontent les listes), Dottrens et Massarenti proposent 2716 mots, qui sont classés en deux listes : la première est classée par ordre alphabétique, chaque mot étant accompagné de son quotient de fréquence et de difficulté orthographique; dans la seconde liste, les mots sont classés par ordre de matières qui correspondent au Vocabulaire vert en usage dans les écoles primaires de Genève, et à l'intérieur de chaque matière dans un ordre croissant de difficulté. À ces 2716 mots, il faut ajouter 23 autres mots donnés en annexe (accent, appareil, appeler...) et 28 mots outils (à, ce, de, elle, en, et, je, etc.).

35 L'importance de cette étude réside pour nous dans le fait que la question de l'acquisition du vocabulaire de la langue française, par des enfants ayant le français comme langue maternelle, est posée : «Il faut [...] que pendant ce laps de temps [les neuf ans que dure la scolarité obligatoire] l'enfant acquière un bagage de mots suffisant pour lui permettre de s'exprimer correctement soit par le langage, soit par ses écrits (1948 ?, p. 1). Cette acquisition doit à la fois être rationnelle (graduée) et aussi convenir à ses besoins (scolaires ou non scolaires) futurs. Les auteurs proposent que les enfants suisses du canton de Genève apprennent un total de 2750 mots le long des « neuf ans de leur scolarité obligatoire ». À la même époque, les élèves de FLE devaient acquérir un minimum de 3 à 4000 mots, en moins d'années et moins d'heures de cours!

\section{Conclusions}

Une première conclusion se dégage de notre présentation : ce sont les professeurs de l'Amérique du Nord qui se sont montrés les plus innovateurs dans ce domaine: la recherche d'un vocabulaire de base ou simplifié servant à améliorer l'apprentissage par des débutants d'une langue étrangère. Cet effort recherche d'un vocabulaire français 
réduit arrive finalement aussi en Europe, comme le montrent les auteurs que nous avons cités ci-dessus, qui élaborent des outils à des fins pédagogiques diverses. Ce sont des auteurs de la Suède, de la Hollande, de la Belgique et de la Suisse qui, les premiers, se rendent compte de l'importance des recherches sur le lexique pour l'enseignement de la langue française en tant que langue étrangère (la Suède, la Hollande), l'enseignement de la langue française en tant que langue maternelle à des fins d'amélioration de la maîtrise de l'orthographe de la part des écoliers du primaire (la Belgique, la Suisse), ou des fins d'acquisition du vocabulaire (la Suisse). Ces auteurs inaugurent une linguistique européenne appliquée à l'enseignement des langues, à base totalement scientifique pourrions-nous dire aussi, puisque la méthode utilisée (la statistique) est couramment acceptée comme une technique de travail qui permet d'arriver à des conclusions "généralisables » et «partagées " par la communauté des chercheurs.

Deuxième conclusion : jusqu'à la publication du Français élémentaire (1954), la France reste à l'écart de cet effort de rénovation, à part quelques exceptions, isolées et individuelles. La linguistique française des années 1930 et 1940 était axée sur d'autres voies de recherche, et surtout, semblait avoir laissé de côté les études sur le langage ordinaire préconisées par Bally et avoir oublié les fortes critiques que F. Brunot avait adressées à l'égard de l'enseignement de la langue maternelle en France, critiques qui semblent avoir à peine entamé des pratiques scolaires déjà séculaires.

Troisième conclusion, ou plutôt constatation : les objectifs marqués dans les travaux sur le vocabulaire que nous avons présentés concernent la lecture et la compréhension des textes écrits, ou bien l'orthographe (l'écrit, encore) dans le cas du français langue maternelle ; il ne s'agissait nullement de rendre l'élève capable de comprendre ou de parler l'oral. C'est-à-dire, les études sur le vocabulaire simplifié s'inscrivent dans le grand problème méthodologique de l'époque, comment accéder aux textes littéraires. Cet accès continuait de se faire en recourant aux morceaux choisis : les élèves, qui avaient suivi normalement dans les deux premiers cours un enseignement de type direct, n'étaient point préparés pour la lecture de ces textes ni pour les exercices associés à elle, les questions-réponses, la rédaction, la dissertation. Au constat de l'absence d'une gradation minimale dans l'assimilation du lexique, s'ajoutaient des pratiques de classe totalement ankylosées : les exercices exigés aux élèves n'étaient pas strictement d'ordre linguistique, ne mettaient pas en jeu la faculté d'expression (à l'oral ou à l'écrit) en langue étrangère, mais par contre un savoir littéraire, stylistique ou rhétorique.

Dans ce contexte, l'irruption du Français élémentaire va permettre de sortir de l'impasse méthodologique de l'époque, en posant le problème d'une façon différente : ce dont il s'agit, pour apprendre une langue étrangère, c'est de bien cerner le vocabulaire de base dans la langue parlée. La France était restée à l'écart de la réflexion sur les vocabulaires simplifiés : c'est cela même qui lui permettra de prendre des distances par rapport aux études et aux propositions antérieures; et, conscient de son retard dans ce terrain, c'est l'État lui-même qui s'investit de façon importante ${ }^{10}$. Nous savons tous que cela entraînera une réorientation des études linguistiques vers la langue orale, la collaboration entre institutions de plusieurs pays et entre équipes formées par des spécialistes de plusieurs domaines, ainsi qu'un profond bouleversement méthodologique dans l'enseignement du FLE. 


\section{BIBLIOGRAPHIE}

BÉNÉDICT G. et HUMBERT J. (1950), « Qu'est-ce que le vocabulaire fondamental ? », Bulletin Pédagogique, 10, p. 200-204.

BESSE H. (1979), « Contribution à l'histoire du français fondamental », Le Français dans le Monde, 148 , p. 23-30.

BONGERS H. (1947), The History and Principles of Vocabulary Control, Wocopi, Woerden.

COHEN M. et al., (1955), Français élémentaire, non? Paris, Éditions Sociales.

COSTE D. (1989), « Aurélien Sauvageot (1897-1988). Un aspect de son œuvre : aux origines de l'élaboration du Français fondamental », Études de Linguistique Appliquée, 75, p. 24-39.

GOUGENHEIM G. (1956), « Français élémentaire ? Oui ou non? », Bulletin de la Faculté des Lettres de Strasbourg, 8-9.

GOUGENHEIM G., MICHÉA P., RIVENC P. et SAUVAGEOT, A., (1956), L'élaboration du Français élémentaire, Paris, Didier.

RIVENC P. (1979), « Le français fondamental vingt-cinq ans après », Le Français dans le Monde, 148, $15-22$.

ROGER G. (1931), « Pour un enseignement plus méthodique et plus scientifique des langues modernes », Les langues modernes.

\section{ANNEXES}

Travaux (études et propositions) concernant les vocabulaires simplifiés au début du XX siècle (présentation chronologique ; domaine : français langue étrangère)

HORNE J. VAN, MORGAN B.Q. (1923-24), « French reading in Secondary Schools, 1918-1923 », Modern Language Journal, VIII, pp. 363-368.

PRESCOTT A. (1923), Le vocabulaire des enfants et des manuels de lecture, Genève, Librairie Kundig.

-S.A., Vocabulaire, Genève, Département de l'Instruction publique de Genève, Imp. P.E. Grivet.

HENMON A.C. (1924), A French Word Bookbased on a count of 400.00 running words,Université de Wisconsin, Madison.

WOOD B.D. (1927), « A Comparative Study of the Vocabularies of Sixteen French textbooks ", Modern Language Journal, XI, 263-289

VANDER BEKE G.E. (1927), A French Word Book, New York, The Mac Millan Company.

BAKER FL. M. (1928), « A tentative vocabulary for first year french students », Journal of educational Research, XVIII, p.369-377.

WEST M. (1929), « Learning a foreign language is largely a matter of acquiring a reading vocabulary ", Language in Education, Longmans Green and Co. 
BLACKBURN M. (1930), « An Analysis of the Vocabulary of two recent French Grammars ", Modern Language Journal, XIV, p. 431-441.

CHEYDLEUR F.D. (1930), (French idiom list based on a running count of 1.183 .000 words, New York, The Mac Millan Company.

SEARS E.K. (1931), « The Vocabularies of Two direct Method French Grammars for Beginners », Modern Language Journal, XV, p. 599-602.

EMERY M.A. (1931), The Composition and Amount of a Minimum Vocabulary for Reading Ungraded French Texts, thèse inédite, Univ. de Chicago.

HAYGOOD J.D. (1937) Vocabulaire fondamental du français, Étude pratique sur l'enseignement des langues vivantes, Paris, Droz.

ARISTIZABAL M. (1938), Détermination expérimentale du vocabulaire écrit pour servir à l'enseignement de l'orthographe à l'école primaire, Louvain.

DUBOIS M.F. (1939), Échelle d'orthographe française, Louvain.

THARP J.B. (1939), Basic French Vocabulary, New York, Henry Holt.

WEST M., BOND O. et LIMPER L.H. (1939), A Grouped-frecuency French Word List based on the French Word Book of Vander Beke, U. Chicago Press.

EATON H.S. (1940), Semantic Frequency List of English, French, German and Spanish,U. Chicago Press.

CAHEN J.-G. (1946), Le vocabulaire de Racine, Paris, E. Droz.

DOTTRENS R. et MASSARENTI D. (1948), Vocabulaire fondamental du français. Contribution à un enseignement rationnel de l'orthographe d'usage, Genève, Delachaux\&Niestlé.

SCHLYTER B. (1951), Centrala ord förrädet i franskan (Vocabulaire central du français), Upsal, Almqvist et Wiksell.

VERLÉE L., (1954) Basis-Woordenboek voor de Franse Taal, Anvers, Sikkel ; Amsterdam, J.M. Meulenhoff.

\section{NOTES}

1. Il faudrait donc dire tout simplement BASIC.

2. Voir à ce sujet Henri Besse (1979) et Daniel Coste (1989), qui situent le projet du FE dans son contexte politique, plus précisément de politique intérieure et de politique culturelle extérieure. Le projet FE, émanant de l'UNESCO, devient l'affaire d'un gouvernement de centre-gauche qui avait remporté la victoire aux élections, certes, mais qui était aux prises d'une forte opposition de droite (gaulliste, $20 \%$ des suffrages) et de gauche (communistes, $26 \%$ des suffrages), comme le rappelle $\mathrm{H}$. Besse (1979: 24-25).

3. Ils manifestent cependant un vif intérêt à son sujet : pour preuve, la très intéressante étude qui porte le titre de «Les origines philosophiques du Basic English », contenue dans le chapitre II (p. 20-25) de L'élaboration du Français élémentaire (1956), étude « qui doit beaucoup à un travail resté inédit de René Michéa ".

4. Les auteurs du FE suivent de près les critiques adressées au Basic par H. Bongers (1947, I, p. 119-134). 
5. Report of the Committee of Twelve of the Modern Language Association of America (D.C. Heath et Co., s.a.), VI + 101 p.).

6. Si nous consultons Bénédict et Humbert (1950) et Haygood ([1937]19482), nous constatons deux différences par rapport à l'Introduction contenue dans Gougenheim et al. (1956). D'une part, la date de l'ouvrage de Vander Beke : 1927 pour ces auteurs, et 1935 pour Gougenheim et al.; d'autre part, chez ces auteurs, c'est le volume XVI de la collection qui correspond à l'ouvrage de Vander Beke, tandis que Gougenheim et al. signalent que c'est le volume XV. Nous avons fait le choix de suivre ces auteurs, car il semble peu logique que le volume XV soit publié en 1935 et le volume XVI en 1929 comme il apparaît chez Gougenheim et al. Il semble aussi peu logique que l'étude sur le français soit publiée après celle de l'espagnol et de l'allemand, langues qui étaient moins étudiées aux USA que le français à l'époque.

7. Il s'agit d'une thèse que J.D. Haygood a présenté à la Faculté des lettres de l'université de Paris. Nous constatons à nouveau des différences entre Bénédict et Humbert (1950, p. 203) et Gougenheim et al. (1956, p. 40). Gougenheim et al. parlent de J.B. Haygood (B. au lieu de Douglas ; il s'agit d'une erreur), et datent son ouvrage de 1936 ; Bénédict et Humbert (1950, p. 203) donnent la date de 1937, que nous avons corroborée par d'autres sources, par exemple Drottens et Massarenti (19482 ?, p. 7).

8. Article publié dans les Cahiers de Pédagogie expérimentale et de psychologie de l'enfant, destinés à faire connaître les recherches et les travaux de l'Institut des Sciences de l'Éducation de l'université de Genève. Nous avons consulté la seconde édition, qui ne porte pas de date, tel que l'avaient constaté Gougenheim et al. en 1956 (page 46, note 6). Le numéro 4 de la nouvelle série, où apparaît cet article, a été publié probablement dans l'année 1948, étant donné que le premier numéro de la nouvelle série date de 1946 et que deux numéros étaient publiés par an. Quant à l'établissement de la date de la première édition, cela comporte un intérêt moindre (la date de création des Cahiers...est de 1934 en tout cas) puisqu'elle a été totalement remaniée.

9. Il s'agit de l'ouvrage suivant : «La pédagogie expérimentale et la langue maternelle ", Cahiers de pédagogie expérimentale et de psychologie de l'enfant, $\mathrm{n}^{\circ}$ 1, 1946.

10. Les responsables ministériels français sont très conscients des retombées linguistiques et socio-culturelles de l'entreprise FE. Le souci de récupérer le retard pris explique le patronage officiel de l'entreprise, l'octroi de fonds, le soutien constant malgré les critiques qui ont été adressées à son égard, la création d'une Commission de travail formée par des spécialistes de divers domaines impliqués ainsi que de son inclusion dans une structure administrative spécialisée et prestigieuse (l'École Normale Supérieure de Saint Cloud).

\section{AUTEUR}

\section{JAVIER SUSO LÓPEZ}

Universidad de Granada 